Classification

Phrsics Abstracts

81.00

\title{
Impact de plaques composites : caractérisation et modèles
}

\author{
Ch. Espinosa et F. Collombet \\ Laboratoire de Mécanique-Physique, U.R.A. C.N.R.S. n 867, Université de Bordeaux I, 351 \\ Cours de la Libération, 33405 Talence Cedex, France
}

(Reçu le 9 avril 1990, révisé le 29 mars et le 29 août 1991, accepté le 6 septembre 1991)

\begin{abstract}
Résumé. - Nous nous intéressons au comportement de plaques minces composites stratifiées à fibres longues et à base de carbone/époxyde ou verre/époxyde. Le travail présenté s'insère dans une étude globale visant la simulation numérique de l'évolution de l'endommagement interne des plaques. Le but de cet article est essentiellement de dégager une relation entre l'état d'endommagement mesuré dans la plaque et l'énergie d'impact absorbée correspondante, à partir des travaux exposés dans la littérature. Pour cela, nous présentons notre analyse des conditions de chargement typiques de la sollicitation d'impact transverse direct: nous différencions les chargements de poinçonnement et de flexion de la plaque sur la localisation, l'initiation et la propagation de la fissuration intralaminaire et du délaminage interlaminaire. Sur la base de cette analyse, nous proposons un modèle phénoménologique d'évolution interactive des dommages tenant compte des modes de chargement et des énergies dissipées, et une identification de l'interaction paramètres expérimentaux/dommages. Nous exposons ensuite notre analyse de modèles de lois de contact par rapport au modèle phénoménologique présenté.
\end{abstract}

\begin{abstract}
We are concerned with the behavior of thin stratified carbon- or glass- epoxy composite plates. The present work is presented as a part of a more wide study carried out which purpose is the numerical simulation of damage evolution within such plates when subjected to a localized transverse low-velocity impact. To establish a correlation between the measured internal damage and the corresponding absorbed energy, from works presented in the literature, is the aim of this one. We first focuse our attention on the typical loading conditions of a direct transverse low-velocity impact. Indeed, we point out the respective role of the impactor/plate contact, indentation and flexion process of the plate on the localization, initiation and propagation of intralaminar cracking and interlaminar delamination. A phenomenological model of interactive evolution of these damages which takes into account the loading and energy absorption process is then proposed, and we identify the interaction between the experimental parameters and the up mentioned damages. We further expose a discussion about some contact law models, and our approach of the loading/structure interaction simulation in terms of the damage phenomenology model presented.
\end{abstract}

\section{Introduction.}

Dans l'industrie aéronautique où elles sont fréquemment utilisées, les structures composites sont parfois soumises à des chocs accidentels de différentes natures : grêlons, particules, mais aussi outils. Lorsque le premier problème de la détection des dommages superficiels est résolu 
à l'échelle de l'observateur, un second problème se pose : celui de la tenue résiduelle de la structure à l'impact. Il s'agit de prévoir les modes de rupture des dégâts occasionnés par l'impact pour empêcher qu'ils n'entraînent la ruine de la pièce.

A l'heure actuelle, les études réalisées sur le sujet ont porté individuellement sur le chargement et/ou le délaminage à l'échelle de la structure, ou sur l'évolution de la fissuration intralaminaire à l'échelle des strates de la plaque. Le but général de cette étude est de déterminer une relation entre un état de dégâts internes, l'histoire du chargement subi par la plaque lors de l'impact, et le comportement post-impact que l'on peut attendre : l'histoire de l'endommagement. Dans cet article, nous proposons un modèle phénoménologique distinguant les chargements de structure de poinçonnement et de flexion, les processus internes de dommages que sont la fissuration intralaminaire et le délaminage interlaminaire, sur la base d'une analyse/synthèse bibliographique. Nous proposons plus particulièrement une interprétation des modes d'interaction des dommages à l'échelle mésostructurelle (de la strate), ainsi que de l'interaction à cette échelle des modes de chargement et des paramètres expérimentaux sur leur localisation, leur initiation et leur propagation.

Du fait de la diversité des travaux concernant le choix du montage, de la métrologie, etc..., ayant servi de base à cet article, les explications qui suivent restent principalement qualitatives. Pour plus de renseignements concernant le cadre d'étude dans lequel certains résultats quantitatifs sont présentés ici, le lecteur devra se reporter à l'article dont ils sont extraits et la référence citée, que nous pensons représentatif parmi une compilation bibliographique étendue.
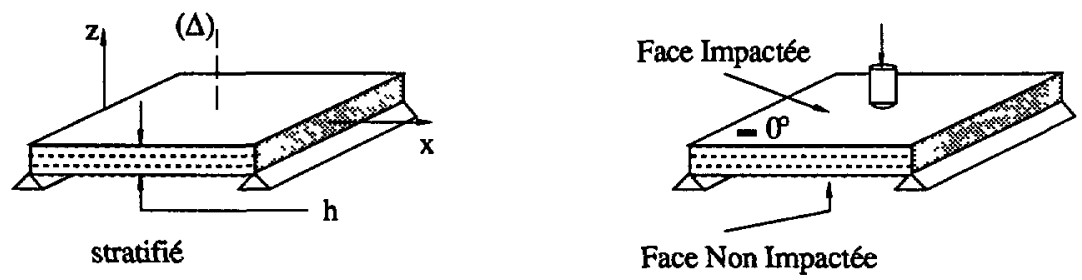

Fig. 1. - Exemple de configuration d'impact. Pour des vitesses intermédiaires, le projectile est souvent une bille tirée suivant $(\Delta) / /(z)$ horizontal.

[Example of impact experiment ; for intermediate velocities, the projectile is often a ball shot following $(\Delta)$ parallel to the horizontal $(z)$ axis.]

Nous nous intéressons ici à des plaques minces stratifiées composites à fibres longues en carbone ou verre et matrice thermodurcissable (époxyde) souvent utilisés pour leur résistance à la rupture élevée, et plus particulièrement aux composites carbone/époxyde dont la résistance aux chocs est deux à trois fois plus faible que celle du verre/époxyde. Néanmoins, l'inspection des dégâts étant plus aisée dans des plaques verre/époxyde, nous nous servirons des observations d'un point de vue qualitatif. Les projectiles usuellement employés sont des billes, des cylindres à tête plate ou hémisphérique de longueur donnée et généralement en acier. Les impacts sont supposés non perforants, directs, centrés, de quelques dizaines de Joules.

L'étude est organisée comme suit :

- la phénoménologie : la sollicitation, l'endommagement, discussion

- étude de modèles de la sollicitation

- conclusions. 


\section{La phénoménologie.}

A l'échelle de l'expérimentateur (le centimètre), la plaque stratifiée est considérée comme une entité qui répond globalement à la sollicitation imposée, dans des conditions expérimentales que l'on veut uniformes : forces d'encastrement, température, taux volumique de défauts initiaux... A cette échelle macroscopique, la théorie classique des stratifiés permet d'assimiler la plaque à une structure globale dont le matériau est homogène, et la sollicitation d'impact à une force ponctuelle, résultante d'un torseur équivalent.

La présence et l'évolution de fissures intralaminaires orientées et de délaminages rend caduque la première approximation; la dépendance de la forme des zones délaminées sur la forme de l'embout du projectile montre la limitation de la seconde. Pour relier l'histoire de la sollicitation d'impact à celle des dommages inter- et intralaminaires, il nous semble donc nécessaire d'étudier chacune d'elles à l'échelle (méso) des strates et de leurs liaisons.

2.1 LA SOllicitation. - L'impact direct se différencie d'un impact indirect ou d'une flexion dynamique par les phénomènes propagatoires différents transmis du projectile à la plaque dans le contact.

A l'instant $t=0 \mathrm{du}$ choc, une onde de compression commence à se propager dans le projectile ainsi qu'une onde tri-dimensionnelle dans la plaque. La forme de l'embout du projectile sur la strate supérieure représente une action de poinçonnement sur la plaque : i.e. un déplacement du projectile dans la plaque sans déplacement de la face arrière ni perforation. Lorsque la composante transverse de cette onde atteint ses appuis, la plaque
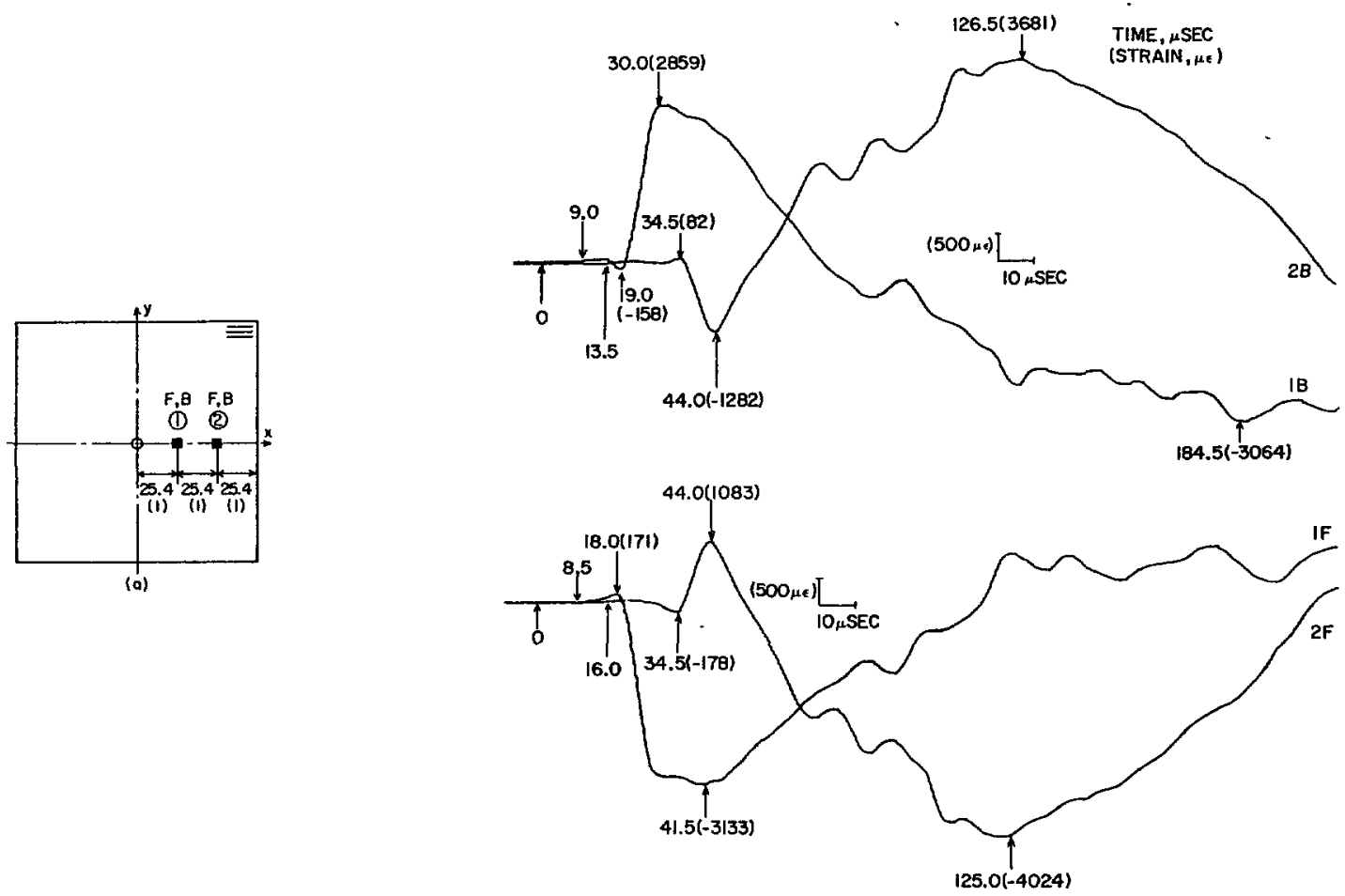

Fig. 2. - Position des jauges $(\mathrm{mm})$ sens fibres $(x)$ sur les faces impactée $(F)$ et non impactée (B) ; enregistrements des déformations à $37,3 \mathrm{~m} / \mathrm{s}$ [1].

[Gage locations (in $\mathrm{mm}$ ) along the fibre direction on the impacted (Front) and non impacted (Back) surfaces; strains records at $37.3 \mathrm{~m} / \mathrm{s}$ [1].] 
amorce un mouvement de flexion qui peut se superposer dans le temps avec l'action de poinçonnement. On schématise le chargement total d'un impact direct par l'enchaînement des quatre stades différenciés ci-dessous : le contact, le poinçonnement seul, la simultanéité poinçonnement/flexion, puis la flexion seule. Cette chronologie apparaît sur les enregistrements ci-dessous (Fig. 3) obtenus pour une plaque verre/époxyde 139,7 $\times 139,7 \mathrm{~mm}^{2}$ (surface utile), $\left[0_{5} / 90_{5} / 0_{5}\right]$, encastrée. Le projectile est en acier, à tête plate de $25,4 \mathrm{~mm}$ de long et $14,175 \mathrm{~g}[1]$.

La quasi-symétrie des signaux montre la prédominance, dans ce cas, d'une onde de flexion transverse dans les deux plans $(x, z)$ et $(y, z)$ de la plaque. Les déformations sont beaucoup plus importantes dans la direction $y$ du plan perpendiculaire aux fibres, et l'onde est plus rapide suivant la direction $x$ longitudinale.

Selon l'auteur de [1], la différence de déformations maximales observée sur les enregistrements de la figure 3 pourrait être due à un endommagement sous les jauges (fissures par exemple). Il suppose que les chutes brutales dans les déformations constatées lors de la propagation de l'onde de "flexion » sont dues à l'initiation puis à la propagation de fissures dans la matrice, voire de délaminage.

De manière générale, la durée relative du poinçonnement dans la durée totale du contact semble proportionnelle à la rigidité de flexion équivalente du système stratifié - conditions aux limites [2]. La durée relative de flexion et le temps total de contact sont inversement proportionnels à cette même rigidité.
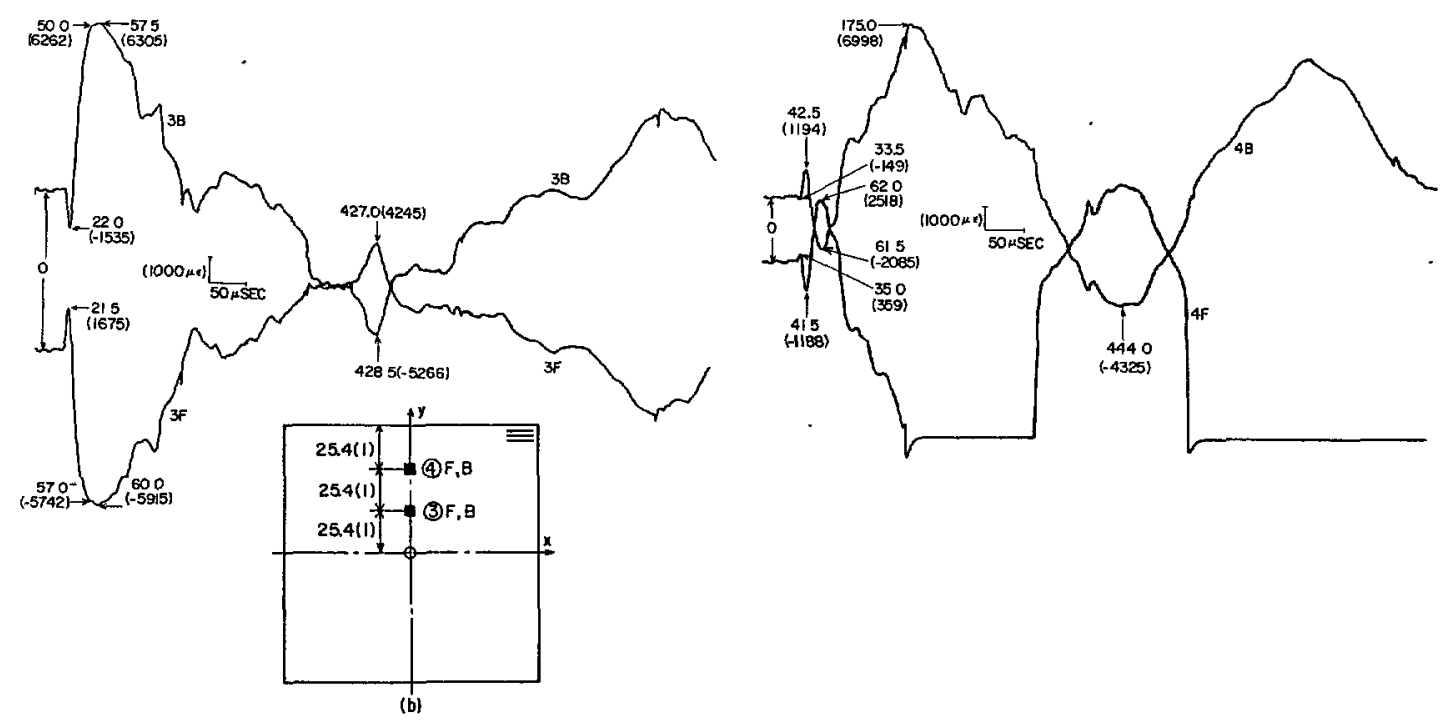

Fig. 3. - Position des jauges (mm) sens travers $(y)$ sur les faces impactée (F) et non impactée (B); enregistrement des déformations à $32,6 \mathrm{~m} / \mathrm{s}$ [1].

[Gage location (in $\mathrm{mm}$ ) in the direction perpendicular to the fibre axis on the impacted (Front) and non impacted (Back) surfaces; strains records at $32.6 \mathrm{~m} / \mathrm{s}$ [1].]

Il a été constaté [1] que des efforts d'impact appliqués pendant un temps plus court créent des pics plus importants dans les déformations et des vitesses de propagation d'ondes plus rapides. Or la durée totale du contact impacteur/plaque dépend non seulement du temps d'aller-retour de l'onde dans l'impacteur, mais aussi des processus de propagation des ondes d'une strate à l'autre. Si le temps de contact est au moins égal à la période de la première vibration de la plaque, les vibrations du système impacteur/plaque ne sont plus négligeables 


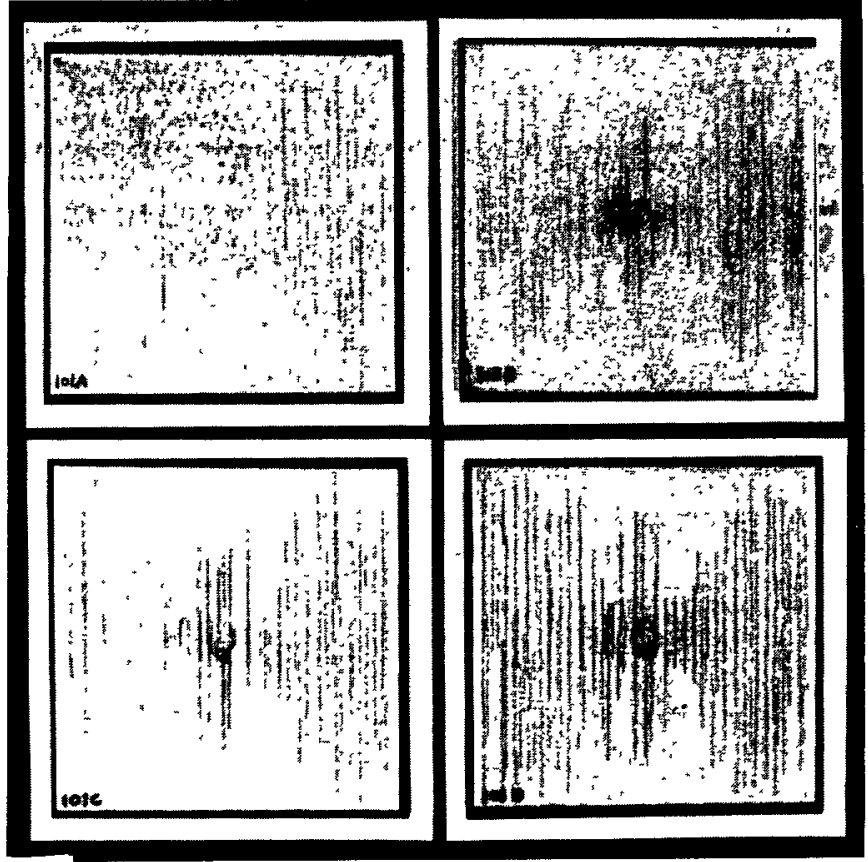

a)

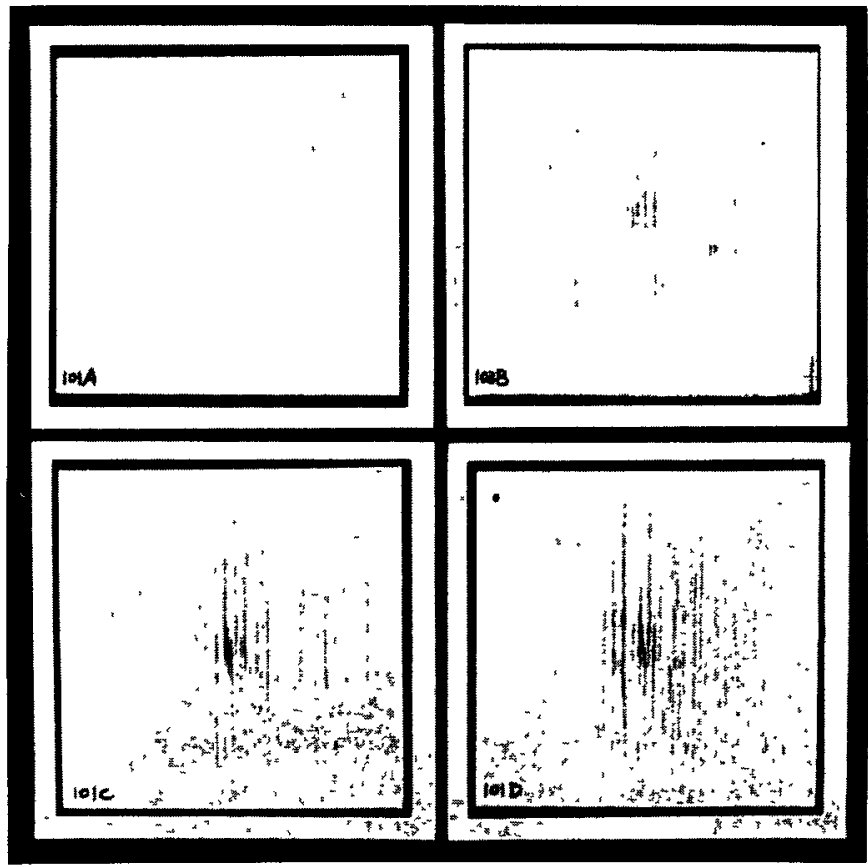

b)

Fig. 4. - Etendue fissurée sur les a) face impactée, b) face non impactée ; quatre plaques verre/époxyde $\left[0_{5}, 90_{5}, 0_{5}\right]$ à énergie croissante ; impacteur cylindrique plat [3].

[Crack pattern on the impacted and non impacted faces of a $\left[0_{5}, 90_{5}, 0_{5}\right]$ glass/epoxy plate at increasing impact energy; flat ended impactor [3].] 
dans l'histoire de la sollicitation. C'est pourquoi il nous semble nécessaire de considérer la plaque en tant que macro-structure constituée par les structures strates et les conditions d'assemblage et de liaisons. Selon nous, l'impact direct de plaque peut être assimilé à une succession de chargements d'une strate sur la suivante à leur échelle.

2.2 L'ENDOMMAGEMENT. - Etant donnée la dimension des fissures intralaminaires et la diversité des orientations des fibres des strates, il n'existe pas, à notre connaissance, de moyen de mesure des étendues fissurées au cours de l'impact. Seuls la découpe multiple des plaques dans les plans transverses à chaque direction de fibre, ou le rétro-éclairage pour des verre/époxyde, après l'impact, permettent de reconstituer les faciès de fissuration respectivement des strates internes et externes.

2.2.1 Analyse post-impact. - Les fissures des faces extérieures de la plaque s'étendent sur des zones symétriques par rapport au point d'impact. Lorsque l'énergie d'impact augmente [3], les fissures se densifient et les zones s'étendent suivant un profil selon nous sinusoïdal marqué sur la face impactée et presque invisible sur la face inférieure (Figs. 4a, b). Les fissures s'initient au milieu de l'épaisseur du pli sous l'action de fortes contraintes de cisaillement, et se propagent jusqu'aux plis adjacents [3].

D'après l'observation des faciès de fissuration après découpe d'autres éprouvettes testées dans des conditions analogues, nous supposons que les zones fissurées des plis intérieurs ont qualitativement les mêmes particularités que celles précitées des plis extérieurs. On observe cependant $[1,4]$ l'absence de fissure au voisinage de $(\Delta)$ dans toute l'épaisseur de la structure (Fig. 6b). Dans le plan de 'chaque pli, il y a une bande non fissurée, appelée «bande génératrice »- «generator strip». - Un schéma descriptif en est donné ci-dessous [1].

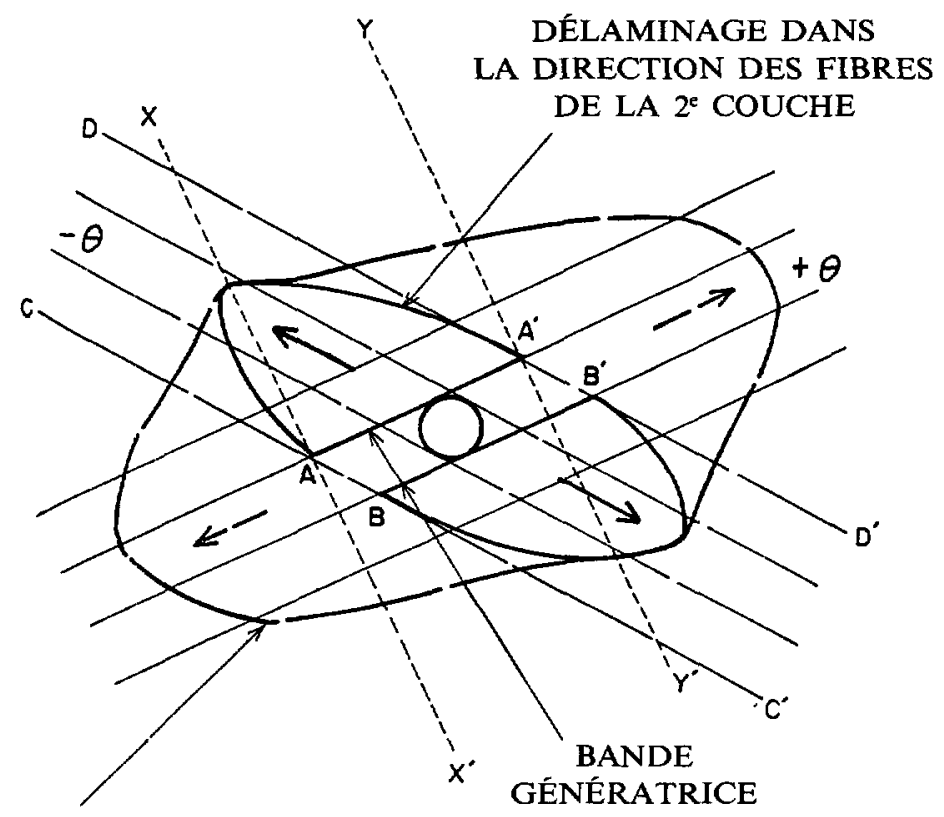

DÉLAMINAGE DANS

LA DIRECTION DES FIBRES

DE LA $3^{\text {c }}$ COUCHE

Fig. 5. - Schématisation de la bande génératrice pour une plaque $\left[\Theta_{5},-\Theta_{5}, \Theta_{5}\right][1]$.

[Scheme of the generator-strips in a plate $\left[\Theta_{5},-\Theta_{5}, \Theta_{5}\right][1]$.] 
Les fissures les plus proches de $(\Delta)$ sont situées à une distance à peu près égale au demirayon du projectile. Elles sont inclinées à $45^{\circ}$, et tendent asymptotiquement vers les interfaces. Les fissures plus éloignées de l'axe $(\Delta)$ sont de moins en moins inclinées puis débouchent perpendiculairement aux interfaces (Fig. 6b) [5, 6].

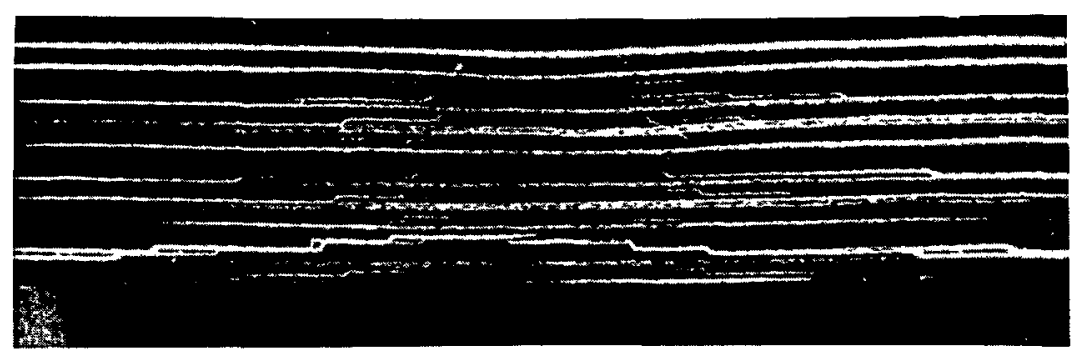

a)

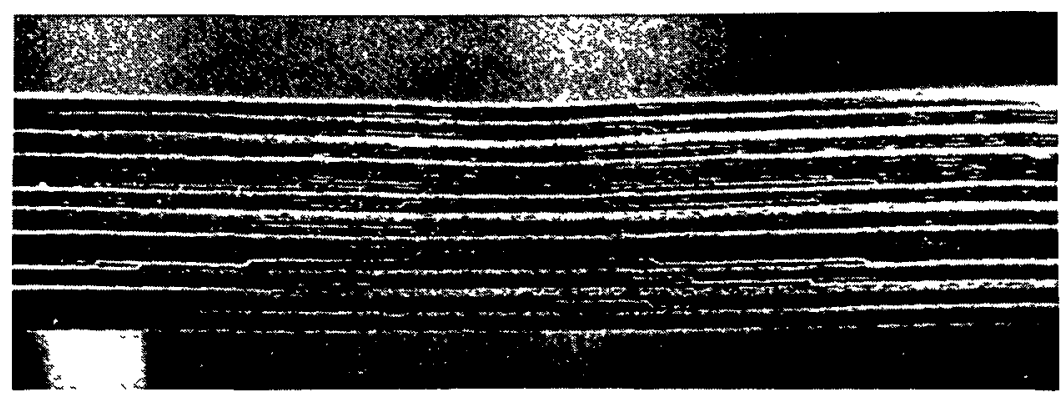

b)

Fig. 6. - Endommagement interne, a) en flexion statique, b) sous impact; plaque quasi-isotrope 48 plis; carbone/époxyde [5].

[Internal damage pattern in a quasi-isotropic 48 ply carbon/epoxy plate under a) static flexion, b) impact [5].]

La vitesse limite d'impact nécessaire à l'apparition des fissures semble être indépendante du type d'impacteur, comme on le voit sur la figure ci-dessous. C'est également le cas de la distance moyenne minimale entre fissures transverses mesurée sur les faces extérieures pour plusieurs tests à énergie variable (Fig. 7) [6].

Il existe des fissures intralaminaires reliant les zones délaminées des interfaces interlaminaires adjacentes (Fig. 6). Pour nous, ces fissures, appelées «criques », sont à distinguer des fissures transverses étudiées ci-dessus.

Le délaminage est un phénomène de físsuration dans le plan, à l'interface de deux plis fissurés dont l'orientation des fibres est différente d'un angle $\Theta$. On note que toute la partie de l'interface située sous la bande génératrice du pli qui la précède n'est pas délaminée. La zone délaminée à chaque interface est constituée de gouttes symétriques par rapport à $(\Delta)$ dont la forme et la taille dépendent de l'état de fissuration dans les plis adjacents. Ces gouttes sont oblongues, orientées suivant la direction des fibres du pli sous l'interface, et d'autant plus allongées que $\Theta$ est plus petit [4]. Leurs extrémités sont plus étroites pour un impacteur hémisphérique et plus larges pour un impacteur plat [6]. Cependant, il est difficile de "voir» dans quelle interface il s'initie: les appareils de mesure non destructive ne permettent de discerner les dommages que lorsqu'ils ont une certaine taille, et le délaminage se propage simultanément dans plusieurs interfaces, à des vitesses différentes (Fig. 8). 
$\circ$ ip $14,175 \mathrm{~g}$

- in $13,275 \mathrm{~g}$

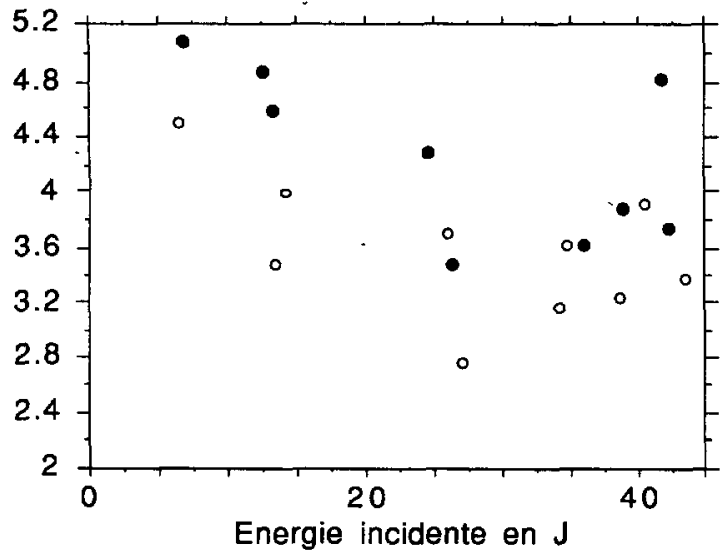

a) (ip) et (ih) de longueur $2,54 \mathrm{~cm}$
○ IP 28,35 g

- IH 27,45 g

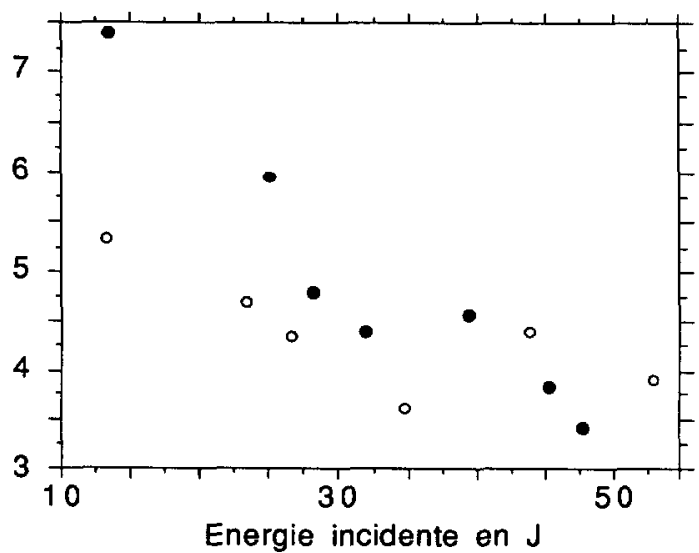

b) (IP) et (IH) de longueur $5,08 \mathrm{~cm}$

Fig. 7. - Distance moyenne entre fissures transverses $(\mathrm{mm})$ sur la face impactée d'une plaque verre/époxyde en fonction de l'énergie incidente pour des impacteurs plats de longueur $2,54 \mathrm{~cm}$ (ip) et $5,08 \mathrm{~cm}$ (IP), et hémisphériques de longueur $2,54 \mathrm{~cm}$ (ih) et $5,08 \mathrm{~cm}$ (IH) [6].

[Mean Transverse Crack Distance (in $\mathrm{mm}$ ) on the impacted surface of a glass/epoxy plate versus incident impact energy for blunt ended $2.54 \mathrm{~cm}$ (ip) and $5.08 \mathrm{~cm}$ (IP) length and hemispherical ended $2.54 \mathrm{~cm}$ (ih) and $5.08 \mathrm{~cm}(\mathrm{IH})$ impactors [6].]
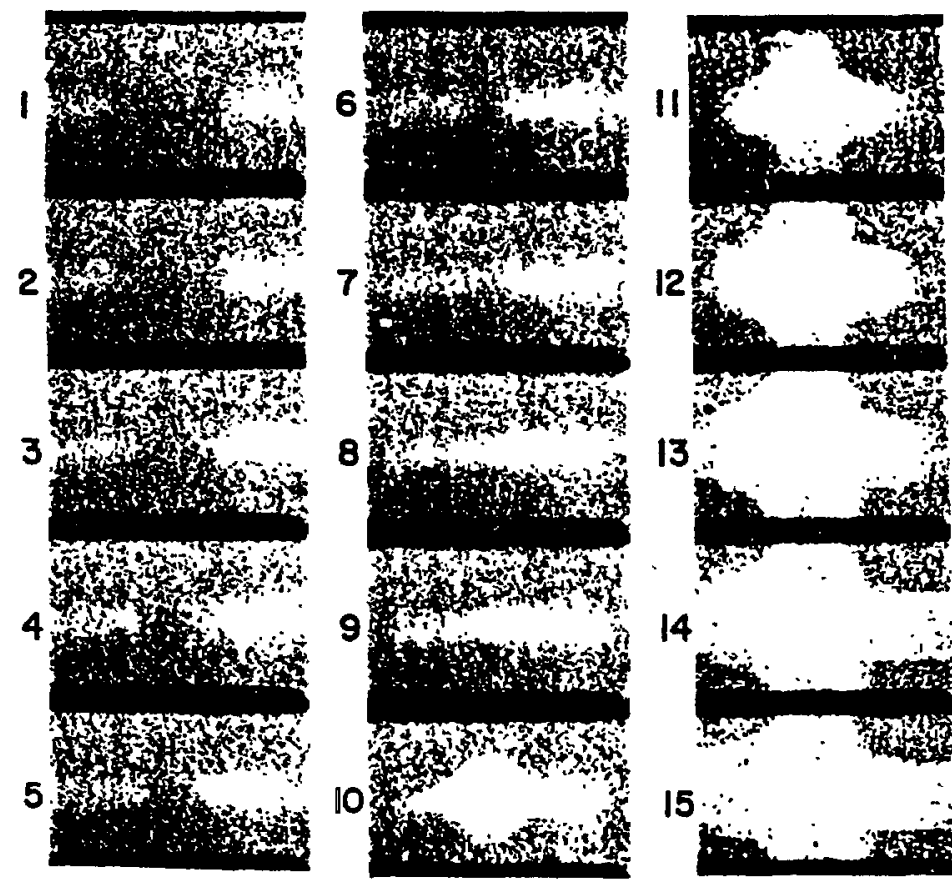

(1-7) Shadows of the impactor are coming towards the center of the target lam nate The impactor veloc ty can be calculated from the shadow velocity (8) The impactor hits the target aminate

(9) Delamination cracks begin to propagate

(11) Both de'amination cracks at the first and second interfaces propagate stmultaneously

(13) The delamination crack at the first interface stops

(15) The delamination crack at the second interface stops
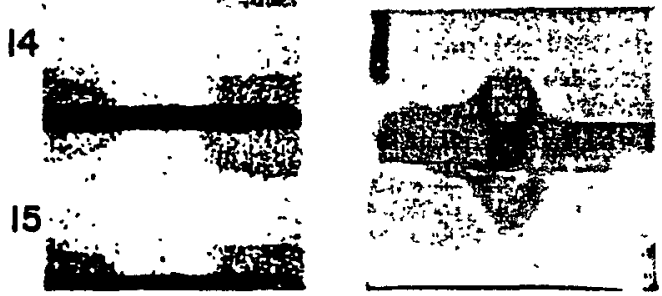

Fig. 8. - Propagation du délaminage; plaque verre/époxyde $\left[0_{5}, 90_{5}, 0_{5}\right]$; projectile plat ; masse $14,175 \mathrm{~g}$, longueur $2,54 \mathrm{~cm}, \phi 9,525 \mathrm{~mm}, 74,5 \mathrm{~m} / \mathrm{s}$ [7].

[Delamination propagation in a glass/epoxy $\left[0_{5}, 90_{5}, 0_{5}\right]$ plate ; blunt ended impactor of $14.175 \mathrm{~g}$ weight, $2.54 \mathrm{~cm}$ length, $9.525 \mathrm{~mm}$ diameter at $74.5 \mathrm{~m} / \mathrm{s}$ [7].] 
2.2.2 Chronologie et énergies. - En vertu de la remarque au début du paragraphe 2.2, nous supposerons respectée la chronologie des dommages, non mesurable pour la fissuration, par la juxtaposition d'états finaux d'endommagement à énergie croissante. Nous définissons leur histoire à partir des étapes du chargement décrites précédemment, en parallèle avec la figure 9 détaillant des paliers d'absorption d'énergie :

- Contact: Dès contact, il y a propagation dans la plaque d'une onde due au choc. Le déplacement du projectile induit une flexion locale du pli supérieur (par compression), i.e. un mouvement de pénétration du projectile dans la plaque entraînant un déplacement des plis supérieurs (proches de la face impactée) sans déplacement de la face non impactée de la structure, et sans perforation, donnant des ruptures de fibres et des fissures traversant l'épaisseur du pli mais superficielles dans la plaque : il y a amorçage du poinçonnement. Cette étape correspond à la région I (Fig. 9).

- Poinçonnement: Le projectile pénètre dans le pli supérieur brisant les fibres et entraînant la propagation des fissures (traversantes) le long des fibres, puis dans les plis sousjacents. La fléchissement local (flexion un point de la plaque) crée des décohésions fibresmatrice, d'autres fissures parallèles aux fibres, et d'imperceptibles zones délaminées dans les interfaces interlaminaires sous le cratère. Les fissures ne sont plus superficielles dans la plaque. Comme on peut s'y attendre, les dommages causés par un impact localisé sont plus importants autour de l'axe $(\Delta)$ de l'impact, et diminuent dans le plan de chaque pli lorsque l'on s'en éloigne [1]. La quantité d'énergie absorbée augmente; on passe dans la zone II (Fig. 9).

- Poinçonnement-flexion: L'onde générée se propage dans la plaque suivant deux "composantes » : une onde dans le plan de chaque pli plus rapide dans la direction des fibres, et dans chaque interface, et une onde dans la direction $(z)$ de l'épaisseur de la structure. On passe de la région II à la région III (Fig. 9). Des zones délaminées sont décelables au C-scan autour de l'axe $(\Delta)$. Les énergies dissipées en poinçonnement et en flexion respectivement diminuent et augmentent. Au cours de ce processus, les fissures se densifient dans les plis extérieurs et la surface totale fissurée s'étend dans le plan. Il apparaît des zones délaminées

\section{- Energie absorbée en $\mathrm{J}$}

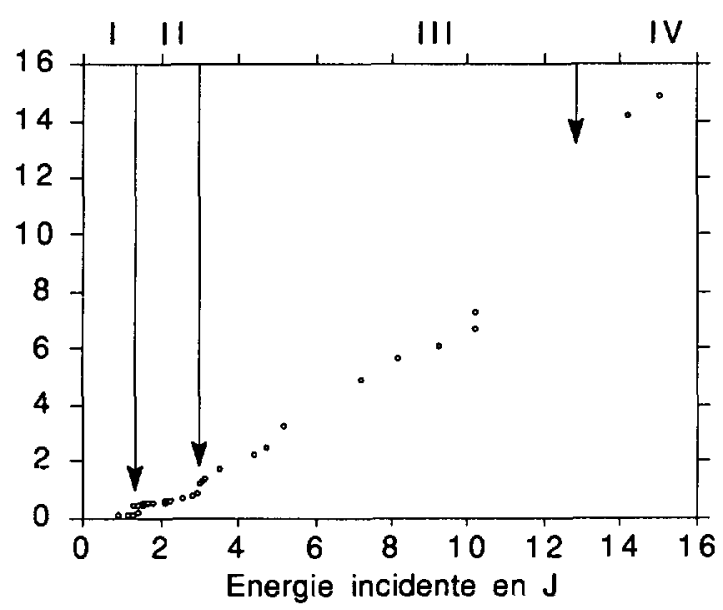

Fig. 9. - « Régions » de dommages en fonction de la quantité d'énergie transmise à une plaque 16 plis en carbone/époxyde [5].

[Damage « regions » versus the absorbed impact energy in a 16 ply carbon/epoxy plate [5].] 
aux interfaces entre les plis d'orientation différente. La proportion relative des ruptures de fibres dans l'endommagement total de la structure reste négligeable pour des énergies d'impact petites devant l'énergie de perforation (quelques Joules). Les phénomènes prépondérants, selon nous, sont la fissuration transverse et le délaminage.

- Flexion : La flexion se poursuit alors que le poinçonnement s'arrête, le projectile étant ralenti. Dans certains cas, la plaque et l'impacteur commencent à vibrer. Nous supposons que les dommages se propagent par cisaillement. Cette étape correspond à la zone III (Fig. 9). L'énergie absorbée augmente par à-coups jusqu'à perforation, ce qui correspond selon nous à l'extension des zones délaminées.

Dans le cas d'un impact unique à énergie élevée, le trou formé sur la face impactée devient plus profond. Au-delà de l'énergie de perforation, les dégâts restent confinés en bord de trou, autour de $(\Delta)$. Seule la Face Non Impactée est réellement endommagée par le projectile qui arrache les fibres en débouchant de la plaque.

La relation endommagement $(d) \div$ énergie fournie $\left(E_{\mathrm{f}}\right)$ n'est pas linéaire. Au-delà d'un seuil, l'accroissement $(\Delta d)$ est une fonction décroissante de $\left(E_{\mathrm{f}}\right)$.

Nous introduisons le schéma qualitatif suivant qui résume selon nous la chronologie observée :

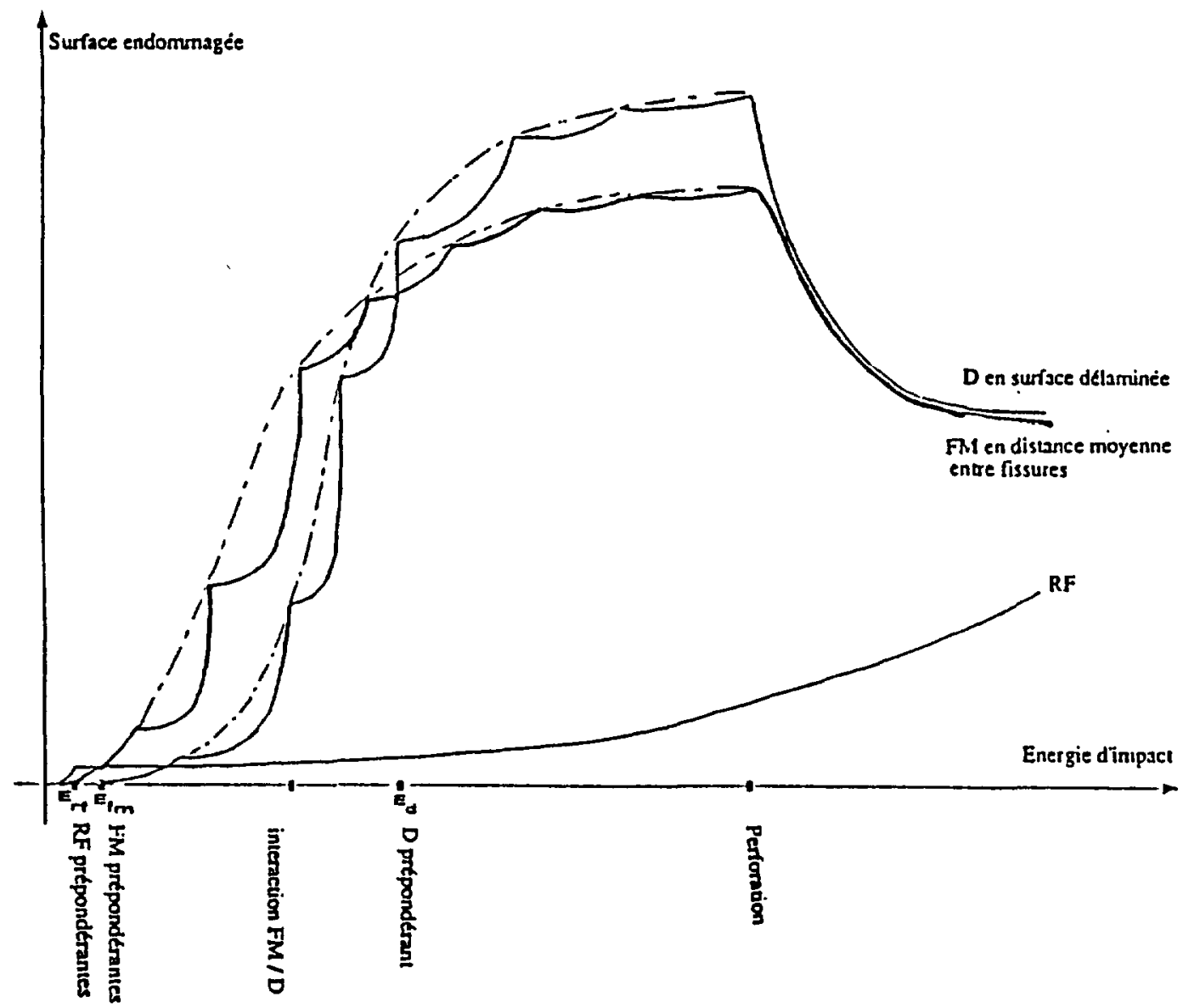

Fig. 10. - Schématisation de l'endommagement en ruptures de fibres, fissuration transverse et délaminage.

[Damage scheme in fibre rupture, transverse cracks and delamination.] 


\subsection{DisCussion.}

2.3.1 Analyse. - Tout comme il existe une distance critique entre fissures transverses dans un pli soumis à une traction statique, nous pensons qu'il existe une distance moyenne critique entre fissures transverses dans chaque pli d'une plaque stratifiée impactée (même intérieur), indépendante de la vitesse d'impact. Il nous semble qu'en fait, le processus de fissuration reflète le comportement du pli en tant que structure dans la plaque, et est particulièrement fonction des rigidités de flexion de chaque pli et de la plaque tout entière. Il ressort de nos observations que le délaminage s'initie avant que la distance moyenne critique entre fissures transverses soit atteinte, même dans les plis adjacents, mais est inexistant à l'interface de plis non fissurés. La forme et l'étendue des zones fissurées dépendent toutes deux essentiellement du type d'impacteur, de l'énergie d'impact, mais aussi des épaisseurs des plis, du type de matériau et des conditions aux limites. L'inverse de cette distance pourrait être une variable représentative de l'endommagement de fissuration, dans une zone localisée et de contour donné du pli.

La fissuration agit à la fois sur l'état et sur l'évolution du délaminage :

- la présence de fissures crée des discontinuités locales de comportement en flexion entre les plis et par conséquent des surcontraintes aux interfaces qui localisent et initient les premiers délaminages. Autour de l'axe $(\Delta)$, le délaminage ne s'initie que dans les zones de l'interface où débouchent et se rejoignent les fissures des deux plis adjacents : la bande génératrice de fissuration dans un pli quelconque borne le délaminage dans l'interface sousjacente ;

- l'ouverture des fissures de la bande génératrice dans chaque pli, et la création successive de ces bandes dans l'épaisseur de la plaque, font respectivement propager les petites zones délaminées ainsi créées, radialement dans les interfaces, et dans l'épaisseur de la plaque. Les surfaces délaminées augmentent géométriquement avec la position de l'interface dans l'épaisseur (vissage hélicoïdal).

Réciproquement, les criques sont la conséquence des délaminages d'interfaces. Il existe donc une forte interaction entre les processus de fissuration et de délaminage, illustrée par la même forme conique des volumes affectés dans l'épaisseur de la structure. Les paramètres essentiels qui contrôlent cette interaction sont ceux qui agissent sur le mode de transmission des efforts du pli sur l'interface et vice-versa, i.e. la séquence d'empilement.

Comme les fissures intralaminaires inclinées, le délaminage est initié et propagé par un cisaillement important provenant du poinçonnement (flexion locale des plis) puis de la flexion de la plaque. La propagation est due à une augmentation des discontinuités de comportement des plis lors de la flexion de la plaque et se fait, en partie, simultanément à la propagation de la fissuration. La forme de la zone délaminée dans chaque interface dépend de la géométrie de l'impacteur, de sa vitesse, et de l'angle $\Theta$ entre les directions des fibres des plis de part et d'autre de l'interface. La surface délaminée semble être une variable représentative de l'endommagement de délaminage subi par la plaque.

Il nous semble donc que le poinçonnement initie à la fois les fissures et le délaminage, et que la flexion de la plaque les propage.

Qu'ils soient rattachés à l'endommagement interne ou à la réponse de structure, l'influence du drapage, de la séquence d'empilement, des conditions aux limites, de l'élancement de la structure, et du type de matériaux constitutifs s'interprète en termes de rigidités de flexion. Lorsque la plaque est rigide à la flexion, l'énergie est dissipée en poinçonnement: les dommages sont précoces mais, à cause de la rigidité importante, se propagent peu et lentement. Ils restent localisés autour du cratère et de $(\Delta)$. Lorsque la plaque est peu rigide le poinçonnement est ralenti. On observe un retard dans l'apparition du délaminage. Cepen- 
dant, comme la flexion est simultanée, il s'étend brusquement et d'autant plus largement que $\Theta$ est grand.

2.3.2 Influence des paramètres expérimentaux. - La répartition des énergies en poinçonnement, flexion de la plaque d'une part, fissuration et délaminage d'autre part provient des interactions de comportement des plis et de la plaque. Nous identifions ci-dessous l'action de chaque paramètre.

La géométrie et la nature des constituants de chaque pli sont prises en compte dans l'expression des rigidités de flexion du pli et de la plaque. Si on peut estimer leur influence sur la localisation et l'étendue des zones fissurées, ce n'est pas le cas, selon nous, sur la densité de fissures du pli ni, à l'échelle de la plaque, sur l'initiation et la propagation de la surface délaminée. La nature des constituants agit sur la fragilité du matériau composite, et l'épaisseur du pli agit sur sa densité de fissures. Nous pensons qu'en augmentant l'épaisseur du pli, on rigidifie le pli à la flexion, mais on le rend plus fragile au poinçonnement. De plus, on accentue la discontinuité de comportement en flexion avec les plis voisins et cela fragilise, selon nous l'interface à la flexion de plaque, ce qui favorise l'extension du délaminage. Cette explication semble corroborer les observations de Liu [4].

Le drapage et la séquence d'empilement sont pris en compte dans l'expression des caractéristiques élastiques de la plaque vierge. Ils conditionnent la valeur du seuil en énergie d'apparition des dommages. Le drapage étant fixé, c'est le choix de la séquence d'empilement qui détermine le degré de discontinuité du comportement en flexion de deux plis consécutifs, en fonction de $\Theta$. Par conséquent, elle agit sur l'évolution de la fragilité de l'interface au cisaillement, et donc sur la forme et l'étendue des zones délaminées [4].

La distance entre appuis ou mords et les conditions aux limites agissent sur la réponse de la structure en vibration, et sur l'influence des phénomènes ondulatoires internes sur le temps et la nature des efforts de contact $(\$ 3)$. Ces paramètres sont à associer au couple (énergie d'impact, impacteur) qui définit indivisément la nature de la sollicitation imposée. Plus l'impacteur est pénétrant, plus la fissuration est dense et localisée autour de l'axe ( $\Delta$ ) dans l'épaisseur de la plaque. L'influence de la géométrie de l'impacteur peut être prise en compte dans l'expression et l'application de la force de contact (\$3).

\section{Etude de la sollicitation.}

La détermination expérimentale du temps de contact passe selon nous par la mesure d'une loi horaire d'application des efforts et la reconnaissance d'une loi de contact. Déjà complexe à l'échelle locale, l'évolution du contact est perturbée par la réponse de la plaque qui subit des cycles de charge-décharge-recharge dans cette zone et du matériau en endommagement. L'apparition de dommages perturbe l'action de la force de contact comme on le voit sur la figure ci-dessous selon les auteurs [5].

\subsection{PRÉSEnTAtion.}

Hertz donña en 1881 une loi décrivant la force résultante $F$ appliquée par un poinçon sphérique élastique sur un demi-espace isotrope élastique [8] :

$$
F=k \alpha^{n} \quad \text { avec } \quad n \approx 3 / 2, \quad \alpha=\alpha(t)=w_{\mathrm{p}}(t)-w(t)
$$

où $w_{\mathrm{p}}$ est le déplacement du projectile, $w$ celui de la plaque au point d'impact; $k$ est un coefficient de contact qui dépend des propriétés de la structure, de celles de la sphère et de son rayon $R_{\mathrm{p}}$ :

$$
k=\frac{4 \sqrt{R_{\mathrm{p}}}}{3}\left[\frac{1-\nu_{\mathrm{p}}^{2}}{E_{\mathrm{p}}}+\frac{1-\nu_{\mathrm{s}}^{2}}{E_{\mathrm{s}}}\right]^{-1}
$$




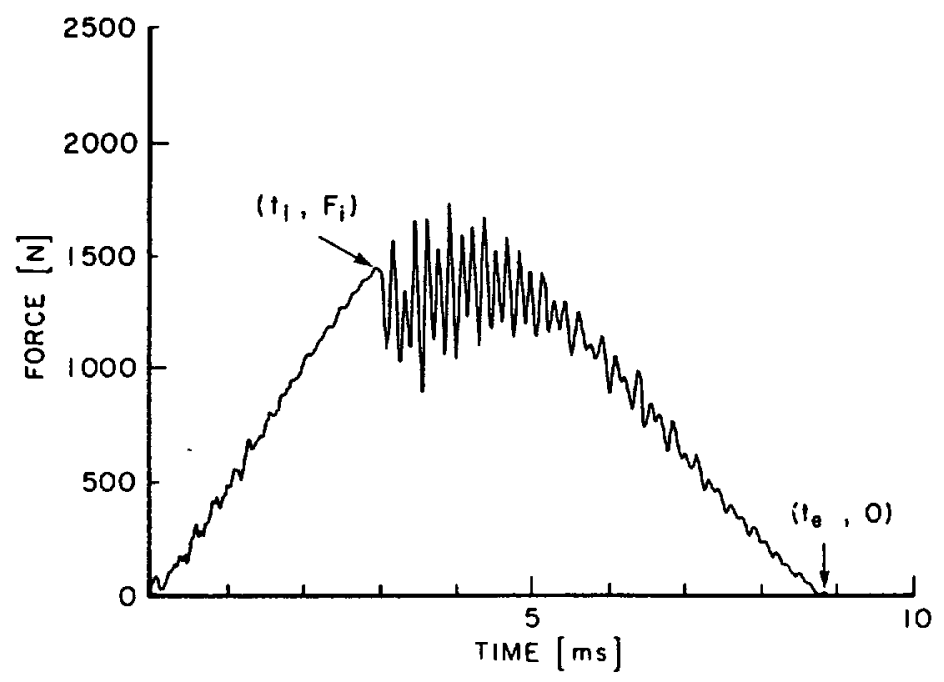

Fig. 11. - Force en fonction du temps sur une plaque carbone/époxyde 16 plis quasi-isotrope simplement posée [5].

[Force versus time in a carbon/epoxy 16 ply quasi-isotropic simply supported [5].]

Les indices $\mathrm{p}$ et $\mathrm{s}$ sont respectivement donnés pour le projectile et la structure.

Cette loi fut étendue par Willis [9] pour le contact d'une sphère avec un demi-espace isotrope transverse. Ces deux versions permettent de traiter l'aspect local de l'impact. Elles ne sont cependant valables que pour les demi-espaces et des matériaux purement élastiques. Dans notre cas, l'épaisseur des plaques est finie, et même pour de faibles chargements, il reste un cratère permanent après décharge. Ceci indique, en particulier, que la courbe de décharge doit suivre un chemin différent de la courbe de charge. Sur la base d'essais statiques, Yang \& Sun [10] ont établi une loi de contact prenant en compte les différents chemins de charge, décharge et recharge. Dans ces essais, différentes distances entre appuis ont été testées. Il semble que le facteur $k$ ci-dessous soit pratiquement constant lorsqu'on fait varier cette dimension pour $n=1,5$.

- Charge

$$
F=k \alpha^{n} \quad k=\frac{4 \sqrt{R_{\mathrm{p}}}}{3}\left[\frac{1+\nu_{\mathrm{p}}^{2}}{E_{\mathrm{p}}}+\frac{1}{E_{2}}\right]^{-1}
$$

où $E_{2}$ est le module de Young transverse du composite et $\alpha$ la variable d'indentation. Le choix de $n$ permet un recalage sur les résultats expérimentaux.

- Décharge (la relation donnée permet de tenir compte du cratère permanent)

$$
F=F_{\mathrm{m}}\left(\frac{\alpha-\alpha_{0}}{\alpha_{\mathrm{m}}-\boldsymbol{\alpha}_{0}}\right)^{q}
$$

où $F_{\mathrm{m}}=$ force à partir de laquelle la décharge s'amorce

$\alpha_{\mathrm{m}}=$ pénétration correspondant à $F_{\mathrm{m}}$

$\alpha_{0}=$ pénétration permanente ou résiduelle dans un cycle de décharge

$q=$ puissance à choisir pour caler le modèle sur l'expérience 
si on pose $s=\frac{F_{\mathrm{m}}}{\left(\alpha_{\mathrm{m}}-\alpha_{0}\right)^{q}}, s$ est une rigidité de décharge, alors on a

$$
F=s\left(\alpha-\alpha_{0}\right)^{q}
$$

- Recharge .

$$
F=k_{1}\left(\alpha-\alpha_{0}\right)^{p}
$$

Selon les auteurs, les valeurs de $n$ et $p$ correspondant le mieux aux résultats expérimentaux sont: $n=3 / 2, p=3 / 2$ pour des verre/époxyde et carbone/époxyde. Pour déterminer $\alpha_{0}$ à partir d'un ensemble de résultats expérimentaux, ils supposent « $s$ » constant quel que soit le trajet de décharge. Il apparaît alors une valeur critique de la pénétration qui semble être un seuil de pénétration élastique, caractéristique du système matériel et de l'impacteur $\alpha_{\mathrm{cr}}=\frac{k}{s}$ au-dessus duquel une déformée permanente apparaît (pénétration résiduelle) ; c'està-dire que $\alpha_{0}=0$ si $\alpha_{\mathrm{m}} \leqslant \alpha_{\text {cr. }}$.

Toujours selon les auteurs, contrairement à l'exposant « $n$ » de la loi de charge pour lequel la valeur $3 / 2$ semble être en bon accord avec les mesures expérimentales, l'exposant $q$ de la loi de décharge varie pour différents diamètres d'impacteur. " $q$ " nous semble donc être révélateur de la forme de l'impacteur, c'est-à-dire de l'évolution de la surface de contact, alors que la longueur conditionne la durée des charge et décharge de contact. Les résultats expérimentaux de [10] montrent que la valeur de $q$ oscille entre 1,5 et 2,5 . Cela peut être dû aux propriétés anisotropes du matériau composite, ou encore à l'influence de la vitesse de décharge. Dans des gammes de vitesses de charge de $20 \mathrm{~N} / \mathrm{s}$ à $200 \mathrm{~N} / \mathrm{s}$, ils notent cependant que l'effet de l'hétérogénéité du matériau semble plus important que celui de la vitesse de mise en charge. Selon les auteurs, cette expression de la force de contact obtenue pour des tests statiques semble convenir à l'étude de l'impact. Ils l'appliquent au seul degré de liberté correspondant au point d'impact, donc ponctuellement [11, 12].

3.2 ANALYSE. - La nature du projectile et l'énergie d'impact sont les seuls paramètres caractérisant la sollicitation transmise à la plaque. C'est la loi de contact choisie qui permet de modéliser leur influence. Il nous semble que la loi déterminée par Yang \& Sun [10] est celle qui prend en compte le plus de paramètres associés à la nature hétérogène et anisotrope du matériau constitutif des plaques. Nous avons constaté que cette nature même conditionnait l'initiation des dommages, la localisation étant due à l'impact proprement dit. La forme de l'impacteur est indirectement prise en compte par l'exposant " $q$ » de la loi. Cependant, dans cette étude présentée au paragraphe 3, l'action réelle de l'impacteur est remplacée par celle de la résultante d'un torseur équivalent, appliqué au point dit « point d'impact ». La force de contact est cette résultante. Les auteurs ramènent le vecteur des efforts extérieurs $\{F\}$ à $\{0, \ldots, 0, f, 0, \ldots, 0\}$, où le seul terme non nul est appliqué au nœud représentant le point d'impact [11]. Ceci, selon nous, permet aux auteurs de s'affranchir de la complexité de représenter la zone de contact, accrue par l'anisotropie du matériau, mais n'est pas réaliste si l'on veut retrouver les processus internes des dommages précédemment exposés. Néanmoins, il nous semble inutile et trop complexe de traduire l'évolution de la surface de contact, et suffisant d'imposer sur plusieurs nœuds une répartition d'efforts au contact. Nous proposons d'imposer, comme conditions initiales, une vitesse identique en ces nœuds au même instant $t=0$ du contact.

Pour rendre compte du rôle prépondérant de l'interface sur la transmission des contraintes (et donc sur la fissuration) d'un pli à l'autre, il est essentiel de traduire le contact particulier 
établi entre plis après délaminage. Pour cela, nous proposons d'utiliser des éléments d'interface autorisant un cisaillement important.

\section{Conclusion.}

Dans cette étude, nous avons choisi de comparer les résultats expérimentaux obtenus pour une gamme étendue d'éprouvettes, de projectiles et de conditions d'essais, afin de tenter de reconstituer un comportement généralisé au choc des plaques minces stratifiées à base de composite à fibres longues.

La localisation, l'initiation et la propagation des processus d'endommagement sont propres à la sollicitation d'impact. La propagation d'ondes propres à un impact direct guide l'extension des surfaces endommagées dans le plan des plis et des interfaces. Nous avons mis en évidence les effets existants selon nous de poinçonnement dans l'initiation et la localisation des dommages, ceux de flexion dans la propagation, et le fait que les proportions relatives de chacun dépendent de la rigidité des plis et de la plaque. Ces proportions relatives agissent sur la valeur des seuils en énergie d'apparition des dommages et leur étendue volumique finale.

L'étude de la phénoménologie nous a permis de dégager les points suivants. La nature des dommages est propre au matériau. Les ruptures de fibres sont en proportion négligeable, sauf pour des énergies très faibles ou de l'ordre de l'énergie de perforation. Pour quantifier la fissuration de matrice, on définit une distance moyenne entre fissures transverses égale à la moyenne arithmétique des distances mesurées. La quantité d'endommagement par délaminage est mesurée par la surface délaminée.

C'est parce qu'une densification des fissures intralaminaires seule permet une déformation importante du stratifié malgré la rigidité de l'empilement, et parce que la fissuration des strates accentue la discontinuité de leur réponse en déformation, que le délaminage s'initie à l'intersection des réseaux de fissures dans les couches adjacentes autour de l'axe $(\Delta)$. Les processus s'induisent mutuellement.

La définition de l'endommagement est liée à celle d'échelle. Pour nous il représente une altération du matériau originel qui se traduit par une chute de ses modules élastiques. Ainsi, le délaminage traduit, selon nous, un endommagement à l'échelle macrostructurelle de la plaque, et une rupture à celle (méso) des strates, modifiant la transmission des efforts à l'interface. C'est en le modélisant à cette échelle qu'il pourra produire des non-linéarités de comportement de structure (flambage). La fissuration, jamais prise en compte à l'échelle de la plaque, est un endommagement à l'échelle des strates. Pour modéliser l'endommagement, nous développons actuellement un modèle de perturbation des modules élastiques des strates composites pour des états de fissuration qui évoluent grâce à un critère de rupture.

\section{Bibliographie}

[1] TAKEDA N., Experimental studies of the delamination mechanisms in impacted fiber reinforced composite plates ; Ph. D. ; University of Florida (1980).

[2] Espinosa Ch. et Collombet F., Qualification de l'endommagement du matériau et des paramètres de structure, étude des contacts; Rapport intermédiaire thèse en cours L.M.P./Aérospatiale; Diffusion restreinte (1989).

[3] LIU D. et Malvern L. E., Matrix cracking in impacted glass/epoxy plates ; J. Compos. Mater. 21 (1987) 594-609.

[4] LIU D., Impact-induced delamination - A view of bending stiffness mismatching; J. Compos. Mater. 22 (1988) 674-692. 
[5] Sјöblom P. O., Cordell T. M. et Hartness J. T., On low-velocity impact testing of composite materials ; J. Compos. Mater. 22 (1988) 31-52.

[6] Takeda N., Sierakowsky R. L. et Malvern L. E., Transverse cracks in glass/epoxy cross-ply laminates impacted by projectiles ; J. Mater. Sci. 16 (1981) 2008-2011.

[7] Takeda N., Sierakowsky R. L., Ross C. A. et Malvern L. E., Delamination-crack propagation in ballistically impacted glass/epoxy laminates; Exp. Mech. (1982) 19-25.

[8] Hertz H., Über die Berührung fester elastischer Körper ; J. die reine angewandte Mathematik 92 (1881) 156-171.

[9] WILLIS J. R., Hertzian contact on anisotropic bodies ; J. Mech. Phys. Solids 14 (1966) 163-176.

[10] YANG S. H. et SUN C. T., Indentation law for composite laminates; ASTM STP 787 (1982) $425-$ 449.

[11] TAN T. M. et SUN C. T., Use of statistical indentation laws in impact analysis of laminated composite plates; J. Appl. Mech. 52 (1985) 6-12.

[12] WU H.-Y. T. et SPRINGER G. S., Impact induced stresses, strains, and delaminations in composite plates; J. Compos. Mater. 22 (1988) 533-560. 
Articles Réguliers 